\title{
Differentiating Glomerular Inflammation from Fibrosis in a Bone Marrow Chimera for Rat Anti-Glomerular Basement Membrane Glomerulonephritis
}

\author{
Cindy Zhou ${ }^{\mathrm{a}}$ Kristie Lou ${ }^{\mathrm{c}}$ Kiana Tatum ${ }^{\mathrm{a}}$ Jeremiah Funk ${ }^{\mathrm{a}}$ Jean Wu \\ Todd Bartkowiak $^{a}$ David Kagan ${ }^{b}$ Yahuan Lou ${ }^{a}$
}

Departments of ${ }^{\mathrm{a}}$ Diagnostic Sciences and ${ }^{\mathrm{b}}$ Internal Medicine, University of Texas Health Science Center at Houston, and ${ }^{\mathrm{C}}$ Department of Biochemistry and Cell Biology, Rice University, Houston, Tex., USA

\section{Key Words}

Glomerulonephritis · Fibrosis · Animal models

\begin{abstract}
Background: Many types of glomerulonephritis (GN) undergo tandem connected phases: inflammation and fibrosis. Fibrosis in human GNs leads to irreversible end-stage disease. This study investigated how these 2 phases were controlled. Methods: Using a rat anti-glomerular basement membrane GN model, we established bone marrow (BM) chimeras between GN-resistant Lewis (LEW) and GN-susceptible Wistar Kyoto (WKY) rats. Glomerular inflammation and fibrosis were compared between chimeras. Results: LEW's BM to WKY chimeras with or without co-transfer of host WKY's T cells were GN-resistant. On the other hand, WKY's BM to LEW (LEWWKY) chimeras developed glomerular inflammation and albuminuria upon immunization. Quantitative analysis showed that the number and composition of inflammatory cells in glom-
\end{abstract}

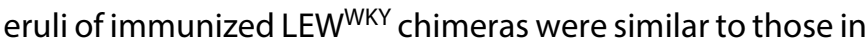
immunized WKY rats at their inflammatory peak. Thus, glomerular inflammation was controlled by BM-derived non-T cell populations. However, unlike WKY rats, LEWWKY rats did not develop fibrosis until the end of experiments (84 days) in spite of persistent inflammation and albuminuria. Conclusion: Inflammation alone was not sufficient to trigger fibrosis, suggesting a critical role of glomerular cells in the fibrotic process. As LEW ${ }^{W K Y}$ chimera allows us to separate glomerular inflammation from fibrosis, this model provides a useful tool to study how fibrosis is initiated following inflammation.

(c) 2015 S. Karger AG, Basel

\section{Introduction}

Many types of glomerulonephritis (GN) such as antiglomerular basement membrane (GBM) GN eventually progress to the glomerular fibrosis phase $[1,2]$. Fibrosis,

\section{KARGER 125}

(2) 2015 S. Karger AG, Base

$0250-8095 / 15 / 0421-0042 \$ 39.50 / 0$

E-Mail karger@karger.com

www.karger.com/ajn
Dr. Yahuan Lou

Department of Diagnostic Sciences, SOD

University of Texas Health Science Center at Houston

Houston, TX 77030 (USA)

E-Mail Yahuan.Lou@uth.tmc.EDU 
which permanently destroys glomerular architecture, is a key step toward the development of end-stage renal disease. Thus, interruption of glomerular fibrosis is an essential therapeutic goal for many types of glomerular diseases [3]. Intensive genetic studies have linked loci/genes to the GN susceptibility in rodent models [47]. However, it remains unclear which loci/genes are specifically associated with the inflammation or fibrosis phase. Kidney and bone marrow (BM) transplantation have been used to identify those cells or genes responsible for each phase [8-10]. However, one potential problem for $\mathrm{BM}$ chimera model is regeneration of the host's BM, which may have led to conflicting results in those studies.

On the other hand, the molecular mechanism underlying fibrosis has been elucidated [11]. TGF- $\beta /$ Smad pathway is critical in mesangial cell activation, as well as differentiation of fibroblasts, which in turn produce extracellular matrix proteins [12-14]. As in many other tissues, the development of renal fibrosis may be an outcome of cross-talk among the cells involved in the 2 phases. Such cross-talk determines the direction toward fibrosis or healing $[15,16]$. More studies are required to understand the cross-talk during the transition from inflammation/injury to initiation of TGF- $\beta /$ Smad pathway. It is necessary to develop models, in which the 2 phases of GN can be studied separately. These models will greatly facilitate the discrimination of aspects of the cross-talk between local cells and inflammatory cells.

In our rat anti-GBM GN model, GN is induced in Wistar Kyoto (WKY) rats by immunization with a T-cell epitope pCol(28-40) from autoantigen collagen $4 \alpha 3$ chain $[17,18]$. T-cell-mediated inflammation is eventually terminated and replaced by progressive fibrosis after day 35 [19]. The Lewis (LEW) rats have identical MHC haplotype and mount a similar T-cell response to $\mathrm{pCol}(28-40)$ as WKY rats. Antigen-presenting cells of LEW rats can effectively present antigens to T cells of WKY rats, and vice versa [20]. However, LEW rats are GN-resistant [19-21]. In this study, BM chimeras between WKY and LEW were created to determine which cells or tissues were responsible for each phase. First, we estimated and minimized the regeneration of the host's $\mathrm{BM}$ to achieve more accurate interpretation of the results. Second, we combined BM chimera with co-transfer of recipients' T cells to determine which BM subpopulation(s) were responsible for GN. Importantly, we were able to segregate glomerular inflammation from fibrosis in a special chimera. Thus, the inflammatory and fibrotic responses were controlled by 2 different cell populations.
Our model may provide another tool to elucidate crossregulation between inflammatory cells and local tissue, which eventually leads to fibrosis.

\section{Methods}

Animals and Construction of BM Chimera

All procedures involving animals in this study was approved by the institutional animal welfare committee. Female WKT or LEW rats (4-6 weeks of age) (Harlan, Indianapolis, IN) were purchased for experiments. BM cells were harvested from donors (6-8 weeks old). Rats (200 g, 6 weeks old) were irradiated with $\gamma$-ray at a total dose of $60-100$ Gy in the animal facility of M.D. Anderson Cancer Center, Houston, and transferred with $15 \times 10^{6}$ $\mathrm{BM}$ cells. The $\mathrm{BM}$ recipients were fed with neomycin-containing water $(2 \mathrm{mg} / \mathrm{ml})$ for 21 days. The chimeras were further immunized with $\mathrm{pCol}(28-40)$ at 28 days after BM grafting. The chimerism was determined by genotyping on peripheral blood leukocytes (PBLs) from the tail vein at day 21, and re-confirmed at the end of the experiment. Briefly, genomic DNA was isolated from various tissue or cells. PCR was performed using primers for 3 polymorphic microsatellites between LEW and WKY rats (D3R201, D13R133, and D1R221) (www.broad.mit.edu/rat/ public). In some cases, T cells were isolated from thymus or lymph nodes of naïve rats with a rat pan-T cell isolation kit (R\&D System, Minneapolis, Minn., USA).

\section{Induction and Evaluation of GN}

Rats were immunized with peptide $\mathrm{pCol}(28-40)(0.15 \mu \mathrm{mol})$ emulsified in CFA, in one hind footpad and at the base of tail. Rats immunized with CFA alone, or without immunization, served as controls. GN was evaluated by albuminuria and renal histopathology. Randomly sampled urine was loaded to $12.5 \%$ SDS-PAGE, and albumin concentration was determined by comparison of a standard bovine serum albumin [20]. A metabolism cage was used to collect urine for 24 hours. A kit from Bio-Rad was used for measuring total urinary creatinine. Urinary albumin to creatinine ratio was calculated. BUN was determined by a colorimetric test kit (Sigma-Aldrich, St. Louse, Mo., USA). Kidney tissues were harvested. Glomerular inflammation was determined by combination of H\&E, PAS and ED1 (i.e. CD68)/RT1B staining, and fibrosis by combination of PAS, collagen $1 \alpha 1($ Coll $1 \alpha 1)$ and a smooth muscle actin (aSMA) staining [22, 23]. Briefly, 50-100 glomeruli were selected on the sections with the same thickness $(3 \mu \mathrm{m})$ for each rat, and 3 subsets of macrophages were counted, or relative Colla1/ aSMA deposition areas were calculated (\% of a glomerulus). The average $>20$ total macrophages/glomerular section was classified as 'inflammation'. Colla1 deposition over 30\%/glomerulus was classified as 'fibrosis'. Total GN score was expressed as a percentage of affected glomeruli. In some case, a portion of kidneys was fixed for transmission electron microscope (EM) following a published method [17].

\section{Antibodies}

PE-labeled anti-rat CD4 (OX35), CD3 (G4.418), CD11b/c (OX-42), and FITC labeled RT-1B (OX6), anti-rat CD4 (OX35), CD90 (Thy-1), and biotin-labeled CD8a (OX8), various rat IgG isotype controls were from Pharmingen BD (San Diego, Calif., 
USA). Monoclonal antibody to ED1 (CD68) was from Santa Cruz Biotech (Dallas, Tex., USA). Antibodies to Colla1 and aSMA were from Abcam (Cambridge, UK) and Sigma-Aldrich (St. Louis, Mo., USA), respectively. FITC-labeled anti-rat IgG were from Southern Biotechnology (Birmingham, Ala., USA). Anti-rat CD32 (D34485) was used for Fc block.

\section{Cell Isolation and Flow Cytometry}

A published method was followed for the isolation of glomeruli-infiltrating leukocytes (GILs) [19]. Briefly, glomeruli were purified to approximately $95 \%$ purity, followed by digestion with collagenase IV and Accutase ${ }^{\mathrm{RT}}$ (ICT, San Diego, Calif., USA) at $37^{\circ} \mathrm{C}$ for $30 \mathrm{~min}$ with periodic stirring. After elimination of digested fragments, the single cells were collected (1,000 rpm, $10 \mathrm{~min})$. Cells were either stained by various pairs of antibodies for flow cytometry (FACSCalibur, BD), or for isolation of a certain population for genotyping. For flow cytometry of GIL populations, stained cells were first gated on FSC vs. SCC plot to enrich leukocyte population. The relative size of each population was calculated as a percentage of total gated cells [20,21]. For depletion or isolation of a certain cell population, magnetic bead-conjugated antibodies were used on a cell sorter (AutoMACS Pro Separator, Miltenyi Biotec, Germany) [19].

\section{Immunofluorescences}

For detection of IgG type autoantibodies to GBM, $3 \mu \mathrm{m}$ frozen sections of frozen renal tissues were incubated with anti-rat IgG FITC. Immunofluorescence with antibodies to RT1B, ED1, CD4, CD8 and CD3 were used for the quantification of various inflammatory cells on kidney sections as described previously using identical thickness of sections (i.e. $3 \mu \mathrm{m}$ ) [20]. Antibody to rat Colla1 and aSMA were used for identifying fibrotic tissues in the glomeruli. All sections were counter-stained by DAPI and observed with a digital fluorescent microscope (Eclipse 80i, Nikon, Tokyo, Japan). Digital images were recorded for evaluation.

\section{In vitro Assay for Antigen Specific T Cell Response}

An established method for measuring $\mathrm{T}$ cell proliferation was adapted to determine antigen specific response of $\mathrm{T}$ cells from immunized rats $[17,20]$. Briefly, T cells were isolated from lymph nodes of immunized rats with a T-cell enrichment column (R\&D System, Minneapolis, Minn., USA). T cells $\left(2 \times 10^{5}\right.$ cells $)$ were incubated with irradiated thymocytes $\left(2 \times 10^{5}\right.$ cells $)$ in 96 -well plates in $200 \mu$ of complete T-cell medium. Peptide pCol(28-40) (1-30 $\mu \mathrm{M})$ was added to each well in triplicate. After $72 \mathrm{~h}$ incubation, the cells were pulsed with ${ }^{3}[\mathrm{H}]$-thymidine, $0.5 \mu \mathrm{Ci} /$ well, for $18 \mathrm{~h}$ (ICN, Costa Mesa, Calif., USA) and harvested onto glass fiber filters. The incorporated radioactivity was measured by MicroBeta TriLux (Perkin Elmer, Waltham, Mass., USA), and the results were expressed as raw count per minute (cpm). Proliferation was also expressed as a stimulation index (experimental cpm at $30 \mu \mathrm{m} / \mathrm{back}$ ground cpm at $0 \mu \mathrm{m})$.

\section{Statistics}

One-way analysis of variance (ANOVA) with a post-hoc Tukey's HSD test was performed for comparison among more than 2 groups. Unpaired t test was used for data comparison between 2 groups. Significances were indicated as follows: ${ }^{*} \mathrm{p}<0.05$, ** $\mathrm{p}<0.01$, and ${ }^{* * *} \mathrm{p}<0.001$.

\section{Results}

\section{Construction and Verification of BM Chimeras}

between GN-Susceptible WKY and GN-Resistant LEW

Chimeras in this study were expressed as a host strain (WKY or LEW) with superscript capital letters indicating $\mathrm{MB}$ donor strain. For example, in chimera WKY ${ }^{\mathrm{LEW}}$, WKY was the host with LEW as the BM donor. The regeneration of host $\mathrm{BM}$ cells in chimeras constructed under various irradiation dosages was determined. Both strains survived irradiation at 60-80 Gy after BM grafting (online suppl. fig. 1a; for all online suppl. material, see www.karger.com/doi/10.1159/000438929). Chimeras, which were irradiated at $60 \mathrm{~Gy}$, showed half of their $\mathrm{BM}$ cells to be the host's origin at day 42 . The majority of lymphocytes isolated from lymph nodes or PBLs of these rats were of the host's origin (online suppl. fig. 1b). Thus, the chimera constructed at $60 \mathrm{~Gy}$ was not suitable for study. On the other hand, in the chimera irradiated with $80 \mathrm{~Gy}$, nearly all BM cells showed donor's genotype (online suppl. fig. $1 \mathrm{~b}$ ). However, a significant portion of $\mathrm{T}$ cells from PBL was still of the host's origin. Since it was the maximum non-lethal dose, 80 Gy was applied for construction of chimeras.

\section{BM-Derived Cells Determine Susceptibility to Glomerular Inflammation}

Twenty WKY ${ }^{\text {LEW }}$ (i.e. WKY with LEW's BM) rats were immunized with pCol(28-40). Immunized $\mathrm{WKY} \mathrm{Y}^{\mathrm{LEW}}$ rats were daily monitored for their albuminuria. Over $72 \%$ of rats did not show any signs of albuminuria (fig. 1a). Histological examination at day 60 demonstrated a complete absence of any inflammation in 14 out of 20, mild inflammation/fibrosis in 4, and significant inflammation/fibrosis in 2 (fig. 1c, e). None of any control WKY ${ }^{\mathrm{LEW}}$, which received CFA only, developed albuminuria or glomerular inflammation (fig. 1c). To further rule out any influence of radiation on susceptibility to GN, 7 auto-BM graft WKY ${ }^{\mathrm{WKY}}$ rats were constructed and immunized. All rats developed severe albuminuria after day 20 (fig. 1a). BNU in these rats reached $181 \pm 27 \mathrm{mg} / \mathrm{dl}$ at day 45 , with a 24 -hour urine albumin/ creatinine ratio of $561 \pm 72$. These rats had to be euthanized due to rapid worsening of their heath condition. Histology showed severe fibrosis in nearly $100 \%$ of glomeruli (fig. 1c, e). Thus, cells derived from donor LEW's BM were responsible for GN resistance in WKY ${ }^{\mathrm{LEW}}$ chimera.

Next, 17 LEW $^{\mathrm{WKY}}$ (i.e. LEW with WKY's BM) rats were immunized with $\mathrm{pCol}(28-40)$. Thirteen rats devel- 

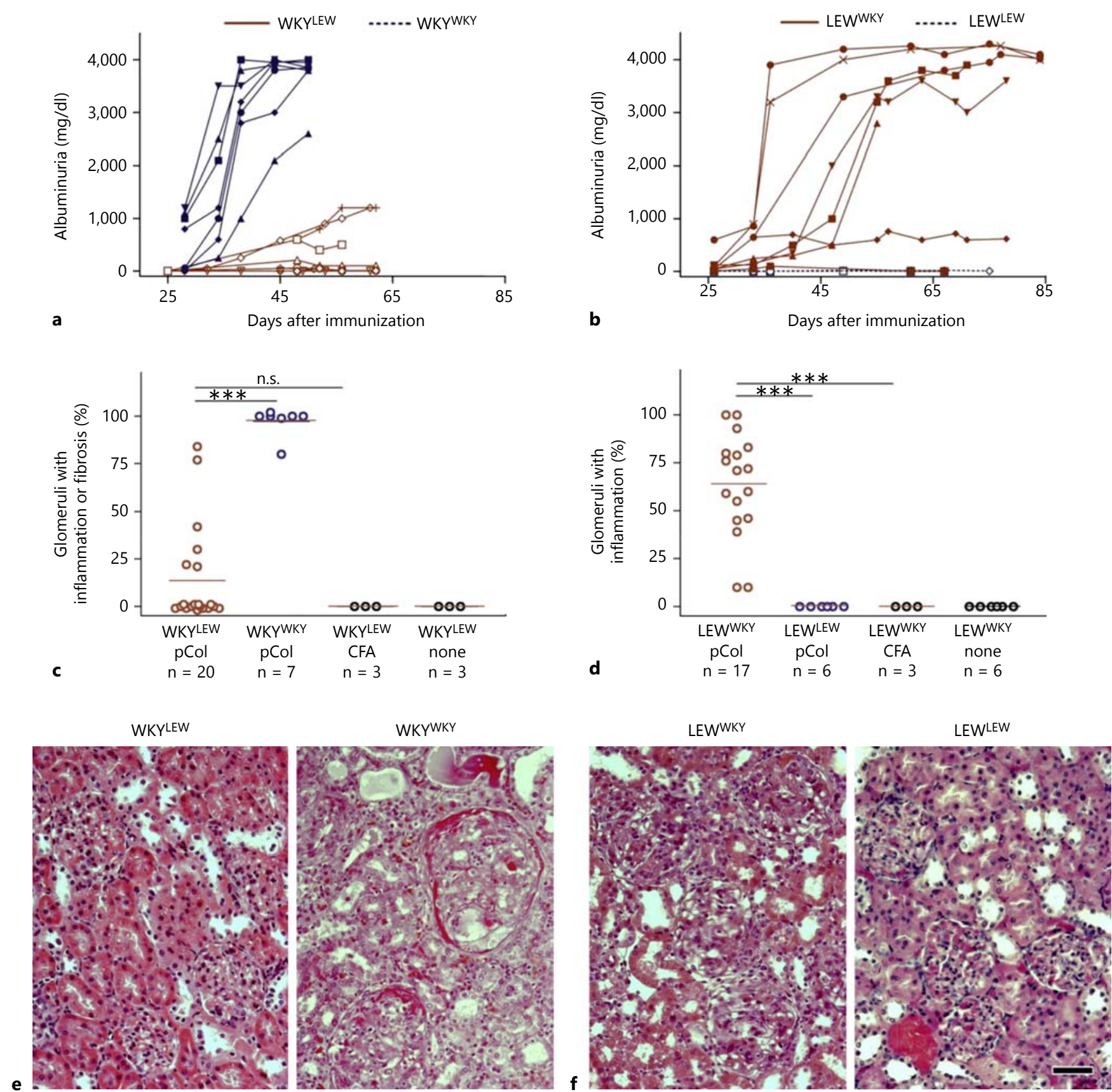

Fig. 1. Development of anti-GBM GN in BM chimeras post immunization with nephritogenic T epitope $\mathrm{pCol}(28-40)$. a, b Time courses of albuminuria in WKY $\mathrm{WEW}^{\mathrm{LE}}$ chimera and related auto-MB graft WKY ${ }^{\mathrm{WKY}}$ controls (a), or in $\mathrm{LEW}^{\mathrm{WKY}}$ and related control $\mathrm{LEW}^{\mathrm{LEW}}(\mathbf{b})$. Albuminuria in each rat is shown separately by different symbols. Closed symbols connected with solid lines are for experimental rats WKY ${ }^{\mathrm{LEW}}$ and $\mathrm{LEW}^{\mathrm{WKY}}$, and open symbols with dashed lines for controls WKY WKY and $\mathrm{LEW}^{\mathrm{LEW}}$. Note that the data for most of WKY ${ }^{\mathrm{LEW}}$ and $\mathrm{LEW}^{\mathrm{LEW}}$ rats are overlapped due to none or insignificant albuminuria. c, d Summaries of GN in WKY ${ }^{\mathrm{LEW}}$ chimera and its controls (c) or $\mathrm{LEW}^{\mathrm{WKY}}$ and their controls (d). Shot horizontal lines represent the mean of each group. One-way ANOVA with post-hoc Tukey's HSD test was performed. *** $\mathrm{p}<0.001 ;$ n.s. $=$ not significant. e, f Representative H\&E staining for each group as indicated. Bar $=50 \mu \mathrm{m}$. 


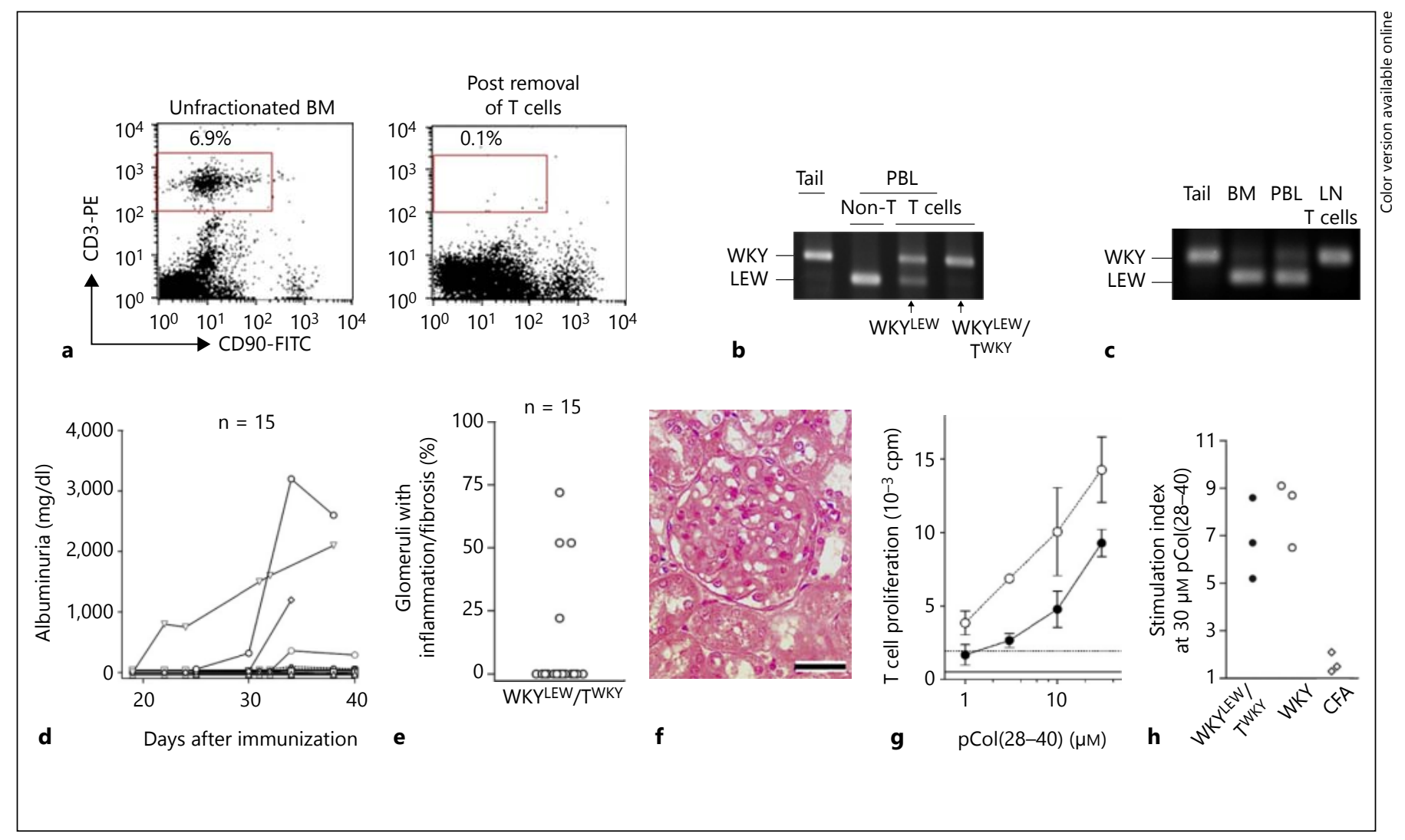

Fig. 2. WKY $\mathrm{LEW}^{\mathrm{LEW}}$ chimeras co-transferred with WKY's $\mathrm{T}$ cells $\left(\mathrm{WKY} \mathrm{YEW}^{\mathrm{LEW}} \mathrm{T}^{\mathrm{WKY}}\right.$ ) do not develop anti-GBM GN after immunization with pCol(28-40). a Flow cytometry shows donor LEW's BM cells before (left) and after (right) removal of T cells. Note a significant reduction in number of $\mathrm{CD}^{+}$cells (gate) post removal of pan-T cells. b PCR genotyping of PBL T cells or non-T cells in $\mathrm{WKY} \mathrm{Y}^{\mathrm{LEW}}$ or $\mathrm{WKY} \mathrm{LEW}^{\mathrm{L}} / \mathrm{T}^{\mathrm{WKY}}$ chimeras. Note that $\mathrm{T}$ cells from a $\mathrm{WKY} \mathrm{Y}^{\mathrm{LEW}} / \mathrm{T}^{\mathrm{WKY}}$ chimera were of WKY's origin, while those of a control WKY ${ }^{\mathrm{LEW}}$ show both genotypes. D3R201 was used as a probe. c Genotyping of T cells of draining lymph node (LN) in $\mathrm{WKY} \mathrm{LEW}^{\mathrm{L}} / \mathrm{T}^{\mathrm{WKY}}$ chimera at day 20 post immunization. d Time course of albuminuria in each $\mathrm{WKY} \mathrm{YEW}^{\mathrm{LEW}} \mathrm{T}^{\mathrm{WKY}}$ rat. Note overlapped lines due to low or none albuminuria in many rats. e Summary of GN scores in immunized $\mathrm{WKY} \mathrm{LEW}^{\mathrm{LEW}} \mathrm{T}^{\mathrm{WKY}}$ chimeras. Each symbol represents an individual rat. f $\mathrm{H} \& \mathrm{E}$ staining shows a representative glomerulus from a WKY ${ }^{\mathrm{LEW}} / \mathrm{T}^{\mathrm{WKY}}$ chimera at day 40. Bar $=50 \mu \mathrm{m}$. g In vitro $\mathrm{T}$ cell proliferation in responding to $\mathrm{pCol}(28-40)$ at day 20 in a representative WKY rat (dashed line) and $\mathrm{WKK} \mathrm{WEW}^{\mathrm{LEW}} \mathrm{T}^{\mathrm{WKY}}$ (solid line). Horizontal lines are background cpm without $\mathrm{pCol}(28-40)$. $\mathbf{h}$ Summary of $\mathrm{T}$ cell proliferation in responding to $\mathrm{pCol}(28-40)$ in different groups. T cell proliferation is expressed as a stimulation index. oped severe albuminuria, which persisted until the end of the experiment (day 84; fig. 1b). Others showed lowlevel but persistent albuminuria. Histology examination from day 70 to 84 showed that 15 out of 17 immunized LEW $^{\mathrm{WKY}}$ developed severe glomerular inflammation (fig. 1d, f). BUN in those rats elevated to $67 \pm 16 \mathrm{mg} / \mathrm{dl}$. Their 24-hour urine albumin/creatinine ratio was $162 \pm$ 20. In contrast, albuminuria or glomerular inflammation was not observed in any LEW ${ }^{\mathrm{WKY}}$, which had received CFA only. In addition, none of 6 auto-BM graft LEW ${ }^{\mathrm{LEW}}$ rats developed albuminuria or GN upon immunization (fig. 1d, f). Thus, BM cells from WKY were responsible for GN in LEW ${ }^{\mathrm{WKY}}$ rats.

\section{T Cells of Host's Origin Does Not Determine GN Susceptibilities in BM Chimera}

The aforementioned experiments did not rule out the involvement of T cells in GN susceptibility because many host's T cells had survived irradiation. We next created chimeras with most $\mathrm{T}$ cells being the host's origin. WKY rats were used as hosts because of their high susceptibility to GN. Any potential pre-T or T cells were depleted from donor LEW's BM cells (fig. 2a). T cells from thymus or lymph nodes from naïve WKY rats were co-transferred with T-cell-depleted LEW's BM cells into irradiated WKY rats. These chimeras with co-transfer of host WKY's T cells were first examined for the origin of myeloid and $\mathrm{T}$
46

Am J Nephrol 2015;42:42-53 DOI: $10.1159 / 000438929$
Zhou/Lou/Tatum/Funk/Wu/Bartkowiak/ Kagan/Lou 
cells at day 30. PBL pan T cells showed a predominantly WKY origin, while other PBLs were of LEW origin (fig. 2b). Thus, myeloid cells in this chimera were from BM of LEW, but T cells were of host WKY's origin. This chimera was designated ' $\mathrm{WKY} \mathrm{Y}^{\mathrm{LEW}} / \mathrm{T}^{\mathrm{WKY}}$ '. Two such chimeras were immunized for determining the origin of activated T cells; nearly all T cells isolated from draining lymph nodes at day 20 were of WKY origin. Next, total 15 $\mathrm{WKY}{ }^{\mathrm{LEW}} / \mathrm{T}^{\mathrm{WKY}}$ were immunized with $\mathrm{pCol}(28-40)$ for observing GN development. Eleven did not show any sign of albuminuria (fig. 2d). Only 4 developed significant albuminuria $(2,000 \mathrm{mg} / \mathrm{dl})$. Eleven rats showed normal glomeruli and 4 with very mild GN at day 45 (fig. 2e, f). Interestingly, mild glomerular fibrosis was observed in those rats with mild GN. Three additional WKY ${ }^{\mathrm{LEW}}$ / $\mathrm{T}^{\mathrm{WKY}}$ rats were immunized for measuring $\mathrm{T}$-cell response at day 20. All 3 showed a strong T-cell proliferative response to $\mathrm{pCol}(28-40)$, which was similar to immunized WKY rats (fig. $2 \mathrm{~g}, \mathrm{~h}$ ). Therefore, LEW myeloid cells, but not WKY's T cells, were responsible for GN resistance in $\mathrm{WKY} \mathrm{LEW}^{\mathrm{L}} / \mathrm{T}^{\mathrm{WKY}}$ chimeras.

\section{Immunized LEW ${ }^{W K Y}$ Chimeras Do Not Develop} Glomerular Fibrosis

Both immunized WKY and auto-BM graft WKY WKY rats developed glomerular fibrosis after 35 days and died after 45 days. However, immunized LEW ${ }^{\mathrm{WKY}}$ rats survived until the end of experiment (84 days) despite persisting severe albuminuria (fig. 1b). Histopathology showed that both $\mathrm{WKY}{ }^{\mathrm{WKY}}$ and $\mathrm{LEW}^{\mathrm{WKY}}$ rats had greatly enlarged glomeruli (fig. 3a). Glomerular fibrosis was then evaluated by PAS staining, and immunofluorescence on Colla1 and aSMA. All 3 methods demonstrated the presence of fibrotic tissue in nearly $100 \%$ of glomeruli in immunized WKY ${ }^{\mathrm{WKY}}$ at day 45. In contrast, fibrotic tissue was not detected in any $\mathrm{WKY}{ }^{\mathrm{WKY}}$ sampled at day 35 (i.e. inflammation peak before entering fibrosis) or $\mathrm{LEW}^{\mathrm{WKY}}$ rats at day 70 (fig. 3a, b). Either Collal deposit area (\%) or its distribution pattern was similar between $\mathrm{WKY}{ }^{\mathrm{WKY}}$ at day 35 and $\mathrm{LEW}^{\mathrm{WKY}}$ at day 70 . On the other hand, a significant number of aSMA ${ }^{+}$fibroblasts was present in Bowman's capsule in $\mathrm{WKY}{ }^{\mathrm{WKY}}$ rat at day 35, but were completely absent in any $\mathrm{LEW}^{\mathrm{WKY}}$ rats at day 70 (fig. 3e). Importantly, $\mathrm{SSMA}^{+}$area/glomerulus in WKY WKY at day 35 was significantly higher than $\mathrm{LEW}^{\mathrm{WKY}}$ at day 70 (fig. 3f). Thus, glomerular fibrosis had been initiated in WKY WKY at day 35 , but not in $\mathrm{LEW}^{\mathrm{W}}$ rats at day 70 . EM study demonstrated similar glomerular damages, including loss of podocyte foot processes in both $\mathrm{LEW}^{\mathrm{WKY}}$ and
WKY at day 35 (fig. 3g). In contrast, WKY at day 45 showed collapse of capillaries with effacement of foot processes.

\section{LEW ${ }^{W K Y}$ Rats Develop Persistent Glomerular}

Inflammation Similar to That in WKY Rats before

Fibrosis

We quantitatively compared GILs between LEW ${ }^{\text {WKY }}$ rats and WKY rats at day 35 (i.e. inflammation peak). Previous analyses on GILs in immunized WKY rats have detected 4 populations: (1) $\mathrm{CD}^{+}{ }^{+} \mathrm{T}$ cell, (2) $\mathrm{CD} 8^{+} \mathrm{T}$ cell, (3) $\mathrm{CD}_{1} 1^{+}$macrophage, and (4) $\mathrm{CD} 11^{+} \mathrm{CD} 8^{+}$cell $[19,21]$. Three immunized LEW ${ }^{\mathrm{WKY}}$ rats with severe albuminuria $(4,000 \mathrm{mg} / \mathrm{dl})$ were selected for isolation of GILs at day 35. Similar to those in the immunized WKY rats, the largest population was $\mathrm{CD}_{1} 1^{+}$phagocyte, followed by $\mathrm{CD}^{+}{ }^{+} \mathrm{CD}^{+}{ }^{+} \mathrm{T}$ cell with $\mathrm{CD} 8^{+} \mathrm{CD}^{+} \mathrm{T}$ cell the smallest (fig. 4a, b) [21]. Relative sizes of the above 3 populations were comparable to those in WKY rats at day 35 (fig. 4c). GILs from $3 \mathrm{LEW}^{\mathrm{WKY}}$ at day 70 with persistent severe albuminuria showed a similar GIL composition (fig. 4c). As expected, flow cytometry did not detect any sizable GIL populations in WKY rats at day 45 due to replacement of inflammation by severe fibrosis (fig. $4 a, c)[20,21]$.

We next examined 3 subsets of infiltrating macrophages (i.e. $\mathrm{ED} 1(\mathrm{CD} 68)^{+}, \mathrm{RT}_{1} \mathrm{~B}^{+}$, and $\left.\mathrm{RT} 1 \mathrm{~B}^{+} \mathrm{ED}^{+}\right)$in situ in $\mathrm{LEW}^{\mathrm{WKY}}$ and WKY groups. $\mathrm{RT}^{+} \mathrm{B}^{+} \mathrm{ED}^{+}$subset was the most populous (fig. 5a, b). No statistically significant differences were observed in the number of all 3 subsets among $\mathrm{LEW}^{\mathrm{WKY}}$ at day 35 and 70 , and WKY ${ }^{\mathrm{WKY}}$ at day 35 (fig. 5b). Distribution patterns of those macrophages were also similar among those groups. They either formed masses within glomeruli or crescent-like clusters in Bowman's capsule (fig. 5a). As expected, only few, if any, macrophages were present in $\mathrm{WKY} \mathrm{WKY}^{\mathrm{W}}$ rats at day 45 (fig. 5a). Fluorescent intensity of RT1B on individual macrophage in glomeruli was measured as an activation marker for macrophages [24]. Glomerular macrophages in both WKY and LEW ${ }^{\mathrm{WKY}}$ groups showed significantly higher density than that of resting residential macrophages (fig. 5c). Thus, the composition, number, distribution pattern, and MHC II expression of macrophages in the inflamed glomeruli of LEW ${ }^{\mathrm{WKY}}$ rats were similar to those in immunized $\mathrm{WKY}{ }^{\mathrm{WKY}}$ rats at day 35 prior fibrosis. $\mathrm{RT}_{1 \mathrm{~B}^{+}}$cells (macrophages and DC) were isolated from glomeruli of LEW ${ }^{\mathrm{WKY}}$ rats (fig. 5d). Genotyping showed that they were of WKY origin (fig. $5 \mathrm{~d}$ ). Thus, glomerular phagocytes in both WKY and LEW had the same genotype of WKY. Finally, immunofluorescence detected GBM-bound autoantibodies in immunized LEW ${ }^{\mathrm{WKY}}$ rats 


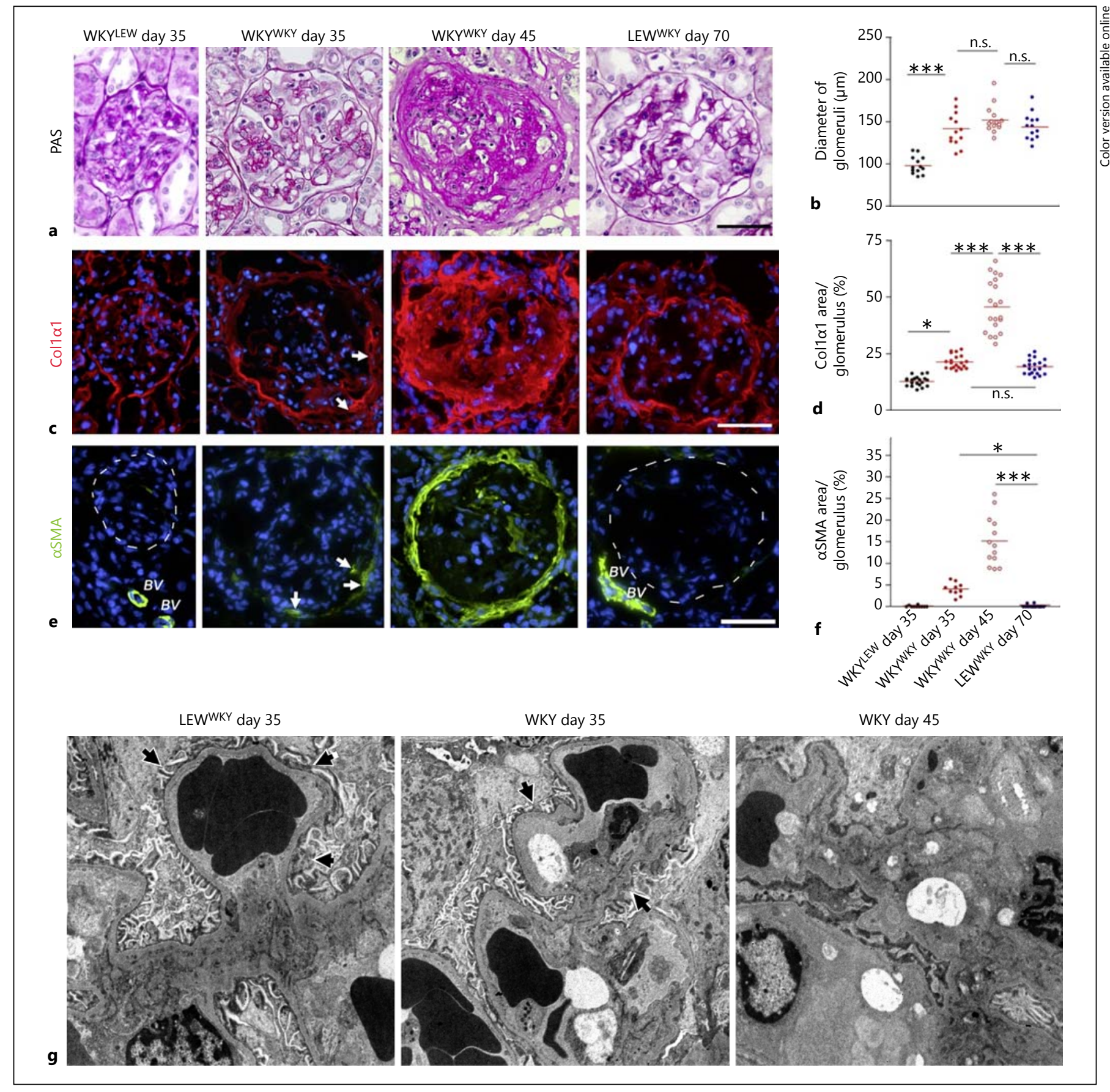

Fig. 3. Absence of fibrotic tissue in glomeruli of $\mathrm{LEW}^{\mathrm{WKY}}$ chimera immunized with $\mathrm{pCol}(28-40)$. a PAS staining patterns in representative glomeruli from different groups and times as indicated at the top of each panel. b Summary of glomerular size in different groups as indicated. c Immunofluorescence shows Collal deposition in glomeruli of different chimeras to reveal fibrotic tissue. Arrows show initiating site of fibrosis. $\mathbf{d}$ Quantitation of Collal deposition in each glomerulus in different groups as indicated. Deposition is expressed as percentage of entire glomerular area. e Immunofluorescence shows aSMA distribution in glomeruli of different groups. Glomerular aSMA ${ }^{+}$cells (arrows) were present in $\mathrm{WKY} \mathrm{WKY}^{\mathrm{WKY}}$ at day 35, but absent in $\mathrm{LEW}^{\mathrm{WKY}}$ rats. Blood vessels (BV), as internal positive control for aSMA. Dash lines outline glomeruli. $\mathbf{f}$ Summary of quantity of aSMA area in each glomerulus in different groups, expressed as percentage of entire glomerular area. One-way ANOVA with post-hoc Tukey's HSD test was performed for $\mathbf{b}, \mathbf{d}$ and $\mathbf{f}$; all 3 with variance $\mathrm{p}<0.0001$. $^{*} \mathrm{p}<0.05$, $* * * \mathrm{p}<0.001 ;$ n.s. $=$ not significant. Red horizontal bars represent the mean of each group. Bars in $\mathbf{a}, \mathbf{c}$ and $\mathbf{e}=50 \mu \mathrm{m}$. $\mathbf{g}$ EM micrographs show glomeruli in different groups of rats as indicated. Arrows indicate abnormal foot processes of podocytes. Note segmental effacement of the processes in WKY at day 45. 

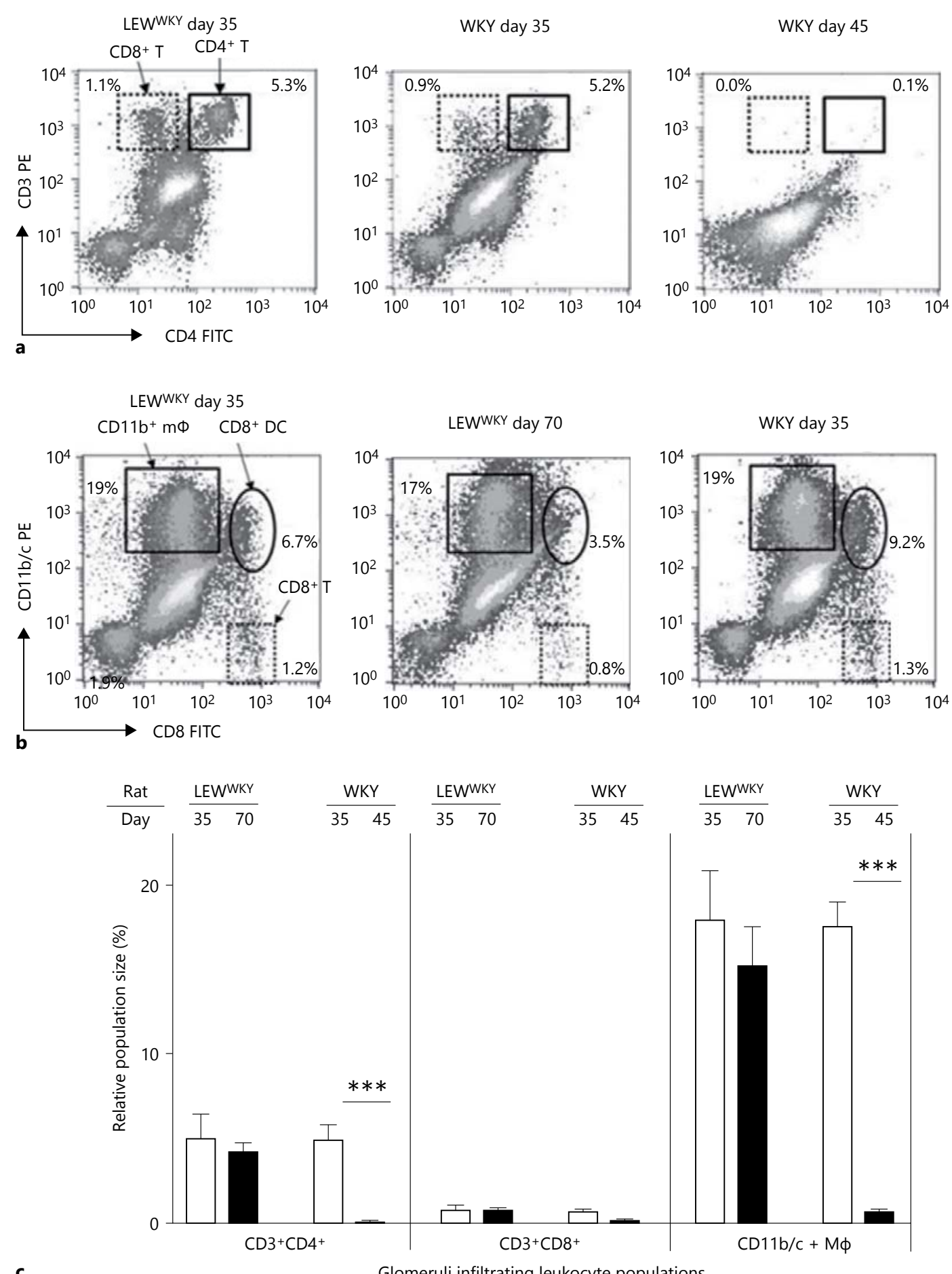

Fig. 4. Flow cytometry on GILs in chimera LEW ${ }^{\mathrm{WKY}}$ and WKY rats post immunization. a Representative density plots for detection of $T$ cells in each group as indicated. A dominant $\mathrm{CD} 4^{+} \mathrm{CD} 3^{+}$population with a minor $\mathrm{CD} 4^{-} \mathrm{CD}^{+}$one is presented in $\mathrm{LEW}^{\mathrm{WKY}}$ day 35 , WKY at day 35, but absent in WKY at day 45. b Representative density plots show $\mathrm{CD} 11^{+} \mathrm{CD} 8^{-}, \mathrm{CD} 11^{+} \mathrm{CD} 8^{+}$, and $\mathrm{CD} 11^{-} \mathrm{CD} 8^{+}$ populations among GILs in chimera LEW ${ }^{\mathrm{WKY}}$ at day 35 and day 70 , as well as in WKY at day 35. c Summary of relative sizes (\%) of 3 GIL populations from different chimera or time as indicated. $\mathrm{n}=3$ for each group, error bars show SD; $M \Phi$, macrophage; $t$ test was performed. ${ }^{* * *} \mathrm{p}<0.001$. 
Fig. 5. Glomerular macrophages and GBMbound autoantibody in chimeras post immunization with $\mathrm{pCol}(28-40)$. a Upper panels show staining for ED1 and Collal. Lower panels are staining for RT1B and ED1 to show 3 macrophage subsets. Clustered macrophages are present in glomeruli of LEW ${ }^{\mathrm{WKY}}$ chimera at day 70 and WKYWKY rat at day 35 , but absent in $\mathrm{WKY}{ }^{\mathrm{WKY}}$ rat at day 45. Arrows show macrophages in Bowman's capsules. Nuclei were counter-stained by DAPI. Note a scarcity of cells in glomeruli of WKY ${ }^{\mathrm{WKY}}$ at day 45 . Bars $=50 \mu \mathrm{m}$. b Summary of glomeruli-infiltrating macrophage subsets in chimeras at various times as indicated. c Surface RT1B expression levels in RT1B ${ }^{+}$ macrophages, expressed as fluorescent intensity, in rats as indicated. Short horizontal bars show the mean for each group. One-way ANOVA with post-hoc Tukey's HSD test was performed for $\mathbf{b}$ and c. d PCR genotyping demonstrates that RT1B ${ }^{+}$GILs were of WKY origin in $\mathrm{LEW}^{\mathrm{WKY}}$ rats (right panel). The left panel shows 2 populations in purified $\mathrm{RT} 1 \mathrm{~B}^{+}$cells. e Immunofluorescence shows GBM-bound IgG autoantibodies in LEW ${ }^{\mathrm{WKY}}$ chimera and WKYWKY control at indicated times. Arrowheads outline a glomerulus for WKY ${ }^{\mathrm{WKY}}$ at day 45 , revealing a large fibrotic tissue surrounding GBM. ${ }^{*} \mathrm{p}<0.05,{ }^{* *} \mathrm{p}<0.01$, $* * * \mathrm{p}<0.001 ;$ n.s. $=$ not significant.

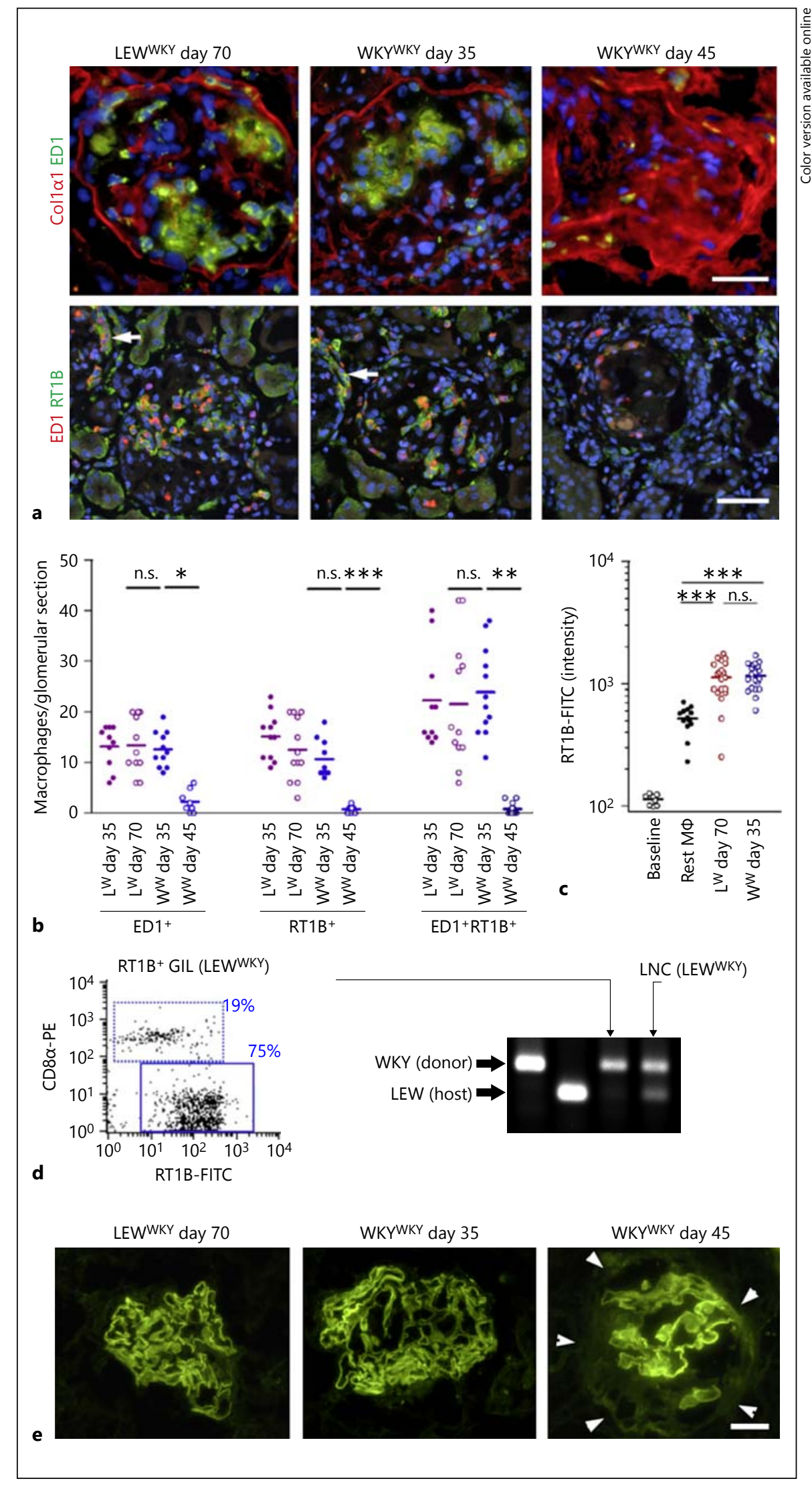

50

Am J Nephrol 2015;42:42-53 DOI: $10.1159 / 000438929$
Zhou/Lou/Tatum/Funk/Wu/Bartkowiak/ Kagan/Lou 
at day 35 and 70 in a pattern similar to that in immunized WKY rats at day 35 (fig. 5e). Thus, T-cell-initiated 'B cell epitope spreading' had also occurred in $\mathrm{LEW}^{\mathrm{WKY}}$ rats [25].

\section{Discussion}

The use of different BM chimeras between WKY and LEW enabled us to demonstrate that glomerular inflammation and fibrosis were sequential events, which were controlled by BM-derived cells and by glomerular cells, respectively. By co-transfer of T-cell-depleted donor's BM cells with host's T cells, we further showed that susceptibility to glomerular inflammation was determined by BM-derived non- $\mathrm{T}$ cell populations. The activation of pathogenic $\mathrm{CD}^{+} \mathrm{T}$ cells is a prerequisite for the initiation of glomerular inflammation as well as anti-GBM antibody production $[17,18,25]$. Activation of T cells was demonstrated in the chimeras (fig. 2). The key question is: Which BM-derived cell populations control susceptibility to glomerular inflammation in parallel with $\mathrm{T}$ cells? We have shown that a BM-derived $\mathrm{RT}_{1 \mathrm{~B}}{ }^{+} \mathrm{CD} 8 \mathrm{aa}^{+} \mathrm{DC}$ like population is associated with GN resistance in LEW rats [19-21]. We have further shown that transfer of LEW's RT $1 B^{+} \mathrm{CD} 8 \alpha \alpha^{+}$cells attenuates severity of GN in WKY rats [26]. Thus, GN resistance in WKY ${ }^{\mathrm{LEW}}$ rats may be due to this DC-like population from LEW's BM. A copy-number polymorphism on the $F c \gamma$ receptor 3 gene has been linked to GN susceptibility [4]. Fc $\gamma$ receptor 3 is expressed in many types of macrophages and DC. This finding suggests an association of certain types of macrophages/DCs with GN susceptibility. By transfer of fractionated BM stem cells, our model may be able to eventually identify the populations, probably macrophages or DC, responsible for GN susceptibility in this rat model.

It has suggested that fibrosis is related to, but independent of, inflammation $[27,28]$. Results in our study supported this concept. Despite persisting glomerular inflammation, LEW ${ }^{\mathrm{WKY}}$ rats did not develop glomerular fibrosis. We quantitatively compared the inflammation between $\mathrm{LEW}^{\mathrm{WKY}}$ and WKY rats. The number, composition and distribution pattern of GILs were very similar to each other. In addition, persistent severe albuminuria in $\mathrm{LEW}^{\mathrm{WKY}}$ rats was evidence of a severe glomerular inflammation. Thus, the absence of fibrosis in $\mathrm{LEW}^{\mathrm{WKY}}$ rats is unlikely due to less severe inflammation. This is supported by the fact that glomerular fibrosis was initiated in a few immunized WKY ${ }^{\mathrm{LEW}}$ rats with mild inflam- mation. However, it is necessary to determine the molecular nature of the inflammation. Because glomerular infiltrating macrophages in both $\mathrm{LEW}^{\mathrm{WKY}}$ and WKY were of WKY origin, one major difference between $\mathrm{LEW}^{\mathrm{WKY}}$ and WKY rats is their glomerular cells. Thus, even the molecular nature of inflammation is different between the 2; this difference is still likely caused by the local glomerular cells. Several previous studies using BM transfer models also supported that glomerular cells may be a determining factor for glomerulosclerosis $[8,9]$. As fibrosis in GN may be a physiological response to irreparable GBM damage and glomerular hemorrhage rather than being an overreaction of healing/repairing process, is it possible that fibrosis will eventually occur in $\mathrm{LEW}^{\mathrm{WKY}}$ rats? Fibrosis in crescentic GN could be associated with both scarring and loss of functional glomeruli because of the failure to precisely realign and heal disrupted basement membranes. If that is the case, the more significant mechanistic question in our model is: What are the glomerular cells and/or expressed genes involved in conveying to the glomerulus and its GBM either protection or susceptibility to the necrotizing and destructive processes?

In human glomerular diseases, regardless of their etiology, glomerular fibrosis is a common consequence leading to end-stage renal diseases $[1,2]$. In many cases, diseases do not enter the fibrosis phase when they are diagnosed. Merely inhibition of ongoing glomerular inflammation may not necessarily lead to normal healing. A method of interruption of fibrotic process is a primary goal for the development of an intervention strategy [3]. Although TGF- $\beta /$ Smad pathway has been a therapeutic target for many years, a successful treatment through inhibition of this pathway has not been developed due to complicated consequences. Investigators have begun seeking new therapeutic targets such as macrophages and stroma cells [29-31]. On the other hand, it remains poorly understood as to how initial damage or inflammation leads to the overactivation of TGF- $\beta /$ Smad pathway in various organs in many human diseases. Elucidation of events that bridge between injury/inflammation and activation of the TGF-b/Smad pathway will provide us with more opportunities to develop intervention strategies for preventing fibrosis de novo, even after the onset of clinical symptoms of a renal disease. From this perspective, our model provides a unique tool for elucidation of such events that occur prior to fibrosis. Our finding in this model is in agreement with many previous studies in several organs (i.e. injured tissues play a critical role in directing re-generation or fibrosis) $[6,30,31]$. Balance in 
expression of a group of genes, including stromal-derived factor-1 receptors, CXCR7 and CXCR4 in sinusoidal endothelial cells post-injury, is the determining factor for regeneration or fibrosis [30]. Expression of certain renal epithelial injury-related molecules leads to renal fibrosis [31]. Many cellular and molecular mechanisms have been explored [32,33]. Importantly, several studies have pointed out that cross-talk among injuries/inflammation and somatic cells leads to this balance $[15,16,33]$. Thus far, it is difficult to interpret such complicated cross-talk and/ or expression patterns using the vitro model alone. Therefore, pairs of $\mathrm{LEW}^{\mathrm{WKY}}$ and WKYWKY rats will facilitate the investigation of such cross-regulations because both rat strains are able to develop similar glomerular inflammation but with opposite consequences (i.e. persistent inflammation versus fibrosis). A systemic comparison of glomerular gene expression patterns between immunized
$\mathrm{LEW}^{\mathrm{WKY}}$ and $\mathrm{WKY} \mathrm{WKY}^{\mathrm{WK}}$ rats at different pathogenic phases will be the first step leading to identification of crosstalk related genes.

\section{Acknowledgments}

This study was supported by NIH R01-DK60029 and R01DK077857 (Y.L.). Histology support was provided by the Pathology Laboratory, University of Texas HSC at Houston. We thank Miss M. Phelps and Dr. C.C. Weng for technical help in creating chimera rats, and Drs. J. McMahon and A. Ross, University of Texas Houston, for reading the manuscript. Dr. J. Hicks, Texas Children Hospital, provided EM technical support.

\section{Disclosure Statement}

The authors do not have any conflict of interest.

\section{References}

1 Meng XM, Nikolic-Paterson DJ, Lan HY: Inflammatory processes in renal fibrosis. Nat Rev Nephrol 2014;10:493-503.

2 Kaissling B, Lehir M, Kriz W: Renal epithelial injury and fibrosis. Biochim Biophys Acta 2013;1832:931-939.

$3 \mathrm{Yu}$ L, Noble NA, Border WA: Therapeutic strategies to halt renal fibrosis. Curr Opin Pharmacol 2002;2:177-181.

4 Aitman TJ, Dong R, Vyse TJ, Norsworthy PJ, Johnson MD, Smith J, Mangion J, RobertonLowe C, Marshall AJ, Petretto E, Hodges MD, Bhangal G, Patel SG, Sheehan-Rooney K, Duda M, Cook PR, Evans DJ, Domin J, Flint J, Boyle JJ, Pusey CD, Cook HT: Copy number polymorphism in Fcgr3 predisposes to glomerulonephritis in rats and humans. Nature 2006;439:851-855.

5 Ijpelaar DH, Schulz A, Aben J, van der Wal A, Bruijn JA, Kreutz R, de Heer E: Genetic predisposition for glomerulonephritis-induced glomerulosclerosis in rats is linked to chromosome 1. Physiol Genomics 2008;35:173181.

6 Liu K, Li QZ, Delgado-Vega AM, et al: Kallikrein genes are associated with lupus and glomerular basement membrane-specific antibody-induced nephritis in mice and humans. J Clin Invest 2009;119:911-923.

7 Reynolds J, Cook PR, Behmoaras J, et al: Genetic susceptibility to experimental autoimmune glomerulonephritis in the Wistar Kyoto rat. Am J Pathol 2012;180:1843-1851.

8 Smith J, Lai PC, Behmoaras J, Roufosse C, Bhangal G, McDaid JP, Aitman T, Tam FW, Pusey CD, Cook HT: Genes expressed by both mesangial cells and bone marrow-derived cells underlie genetic susceptibility to cres- centic glomerulonephritis in the rat. J Am Soc Nephrol 2007; 18:1816-1823.

9 Aben JA, Hoogervorst DA, Paul LC, Borrias MC, Noble NA, Border WA, Bruijn JA, de Heer E: Genes expressed by the kidney, but not by bone marrow-derived cells, underlie the genetic predisposition to progressive glomerulosclerosis after mesangial injury. J Am Soc Nephrol 2003;14:2264-2270.

10 Schreiber A, Xiao H, Falk RJ, Jennette JC: Bone marrow-derived cells are sufficient and necessary targets to mediate glomerulonephritis and vasculitis induced by anti-myeloperoxidase antibodies. J Am Soc Nephrol 2006;17:3355-3364.

11 Eddy AA: Molecular basis of renal fibrosis. Pediatr Nephrol 2000;15:290-301.

12 Border WA, Okuda S, Languino LR, Sporn MB, Ruoslahti E: Suppression of experimental glomerulonephritis by antiserum against transforming growth factor beta 1 . Nature 1990;346:371-374.

13 Gaedeke J, Peters H, Noble NA, Border WA: Angiotensin II, TGF- $\beta$ and renal fibrosis; in Wolf G (ed): The Renin-Angiotensin System and Progression of Renal Diseases. Basel, Karger, 2001, vol 135, pp 153-160.

14 Yanagita M: Inhibitors/antagonists of TGF- $\beta$ system in kidney fibrosis. Nephrol Dial Transplant 2012;27:3686-3691.

15 Wynn TA, Ramalingam TR: Mechanisms of fibrosis: therapeutic translation for fibrotic disease. Nat Med 2012;18:1028-1040.

16 Lin SL, Chang FC, Schrimpf C, Chen YT, Wu CF, Wu VC, Chiang WC, Kuhnert F, Kuo CJ, Chen YM, Wu KD, Tsai TJ, Duffield JS: Targeting endothelium-pericyte cross talk by inhibiting VEGF receptor signaling attenuates kidney microvascular rarefaction and fibrosis. Am J Pathol 2011;178:911-923.

17 Wu J, Hicks J, Borillo J, Glass WF 2nd, Lou YH: CD4(+) T cells specific to a glomerular basement membrane antigen mediate glomerulonephritis. J Clin Invest 2002;109:517-524.

18 Robertson J, Wu J, Arends J, Glass W 2nd, Southwood S, Sette A, Lou YH: Characterization of the T-cell epitope that causes antiGBM glomerulonephritis. Kidney Int 2005; 68:1061-1070.

$19 \mathrm{Wu}$ J, Zhou C, Robertson J, Weng CC, Meistrich ML, Tailor RC, Lou YH: Identification of a bone marrow-derived CD8a + dendritic cell-like population in inflamed autoimmune target tissue with capability of inducing T cell apoptosis. J Leukoc Biol 2010;88:849-861.

20 Robertson J, Wu J, Arends J, Zhou C, Adrogue $\mathrm{H}$, Chan JT, Lou Y: Spontaneous recovery from early glomerular inflammation is associated with resistance to anti-GBM glomerulonephritis: tolerance and autoimmune tissue injury. J Autoimmun 2008;30:246-256.

21 Zhou C, Wu J, Robertson J, Bartkowiak T, Parker K, McMahon J, Lou YH: Natural recovery from antiglomerular basement membrane glomerulonephritis is associated with glomeruli-infiltrating $\mathrm{CD} 8 \mathrm{a}+\mathrm{CD} 11 \mathrm{c}+\mathrm{MHC}$ class II+ cells. Am J Nephrol 2011;34:519528.

22 Rossert J, Terraz C, Dupont S: Regulation of type I collagen genes expression. Nephrol Dial Transplant 2000;15(suppl 6):66-68.

23 Boukhalfa G, Desmoulière A, Rondeau E, Gabbiani G, Sraer JD: Relationship between alpha-smooth muscle actin expression and fibrotic changes in human kidney. Exp Nephrol 1996;4:241-247. 
24 Mosser DM: The many faces of macrophage activation. J Leukoc Biol 2003;73:209212.

$25 \mathrm{Wu}$ J, Arends J, Borillo J, Zhou C, Merszei J, McMahon J, Lou YH: A self T cell epitope induces autoantibody response: mechanism for production of antibodies to diverse glomerular basement membrane antigens. J Immunol 2004;172:4567-4574.

$26 \mathrm{Wu}$ J, Zhou C, Robertson J, Carlock C, Lou YH: Peripheral blood CD8a $\alpha+C D 11 c+$ MHCII $+\mathrm{CD} 3$ - cells attenuate autoimmune glomerulonephritis in rats. Kidney Int 2014;85: 1078-1090.
27 Paz Z, Shoenfeld Y: Antifibrosis: to reverse the irreversible. Clin Rev Allergy Immunol 2010;38:276-286.

28 Ding BS, Cao Z, Lis R, Nolan DJ, Guo P, Simons M, Penfold ME, Shido K, Rabbany SY, Rafii S: Divergent angiocrine signals from vascular niche balance liver regeneration and fibrosis. Nature 2014;505:97-102.

29 Schuppan D, Kim YO: Evolving therapies for liver fibrosis. J Clin Invest 2013;123:18871901.

30 Humphreys BD, Xu F, Sabbisetti V, Grgic I, Naini SM, Wang N, Chen G, Xiao S, Patel D, Henderson JM, Ichimura T, Mou S, Soeung S,
McMahon AP, Kuchroo VK, Bonventre JV: Chronic epithelial kidney injury molecule-1 expression causes murine kidney fibrosis. J Clin Invest 2013;123:4023-4035.

31 Duffield JS: Cellular and molecular mechanisms in kidney fibrosis. J Clin Invest 2014; 124:2299-2306.

32 Brosius FC 3rd: New insights into the mechanisms of fibrosis and sclerosis in diabetic nephropathy. Rev Endocr Metab Disord 2008;9: 245-254.

33 Lee SB, Kalluri R: Mechanistic connection between inflammation and fibrosis. Kidney Int Suppl 2010;119:S22-S26. 